\title{
VALORACIÓN DEL IMPACTO SOCIOECONÓMICO DE LA VARIABILIDAD CLIMÁTICA EN PESCA Y TURISMO ${ }^{1}$
}

\section{SOCIO-ECONOMIC IMPACT ASSESSMENT OF CLIMATE VARIABILITY IN FISHERIN AND TOURISM}

\author{
Mary Luz Moreno Díaz*
}

\begin{abstract}
RESUMEN
El objetivo del presente artículo es analizar las investigaciones que se han desarrollado, con el fin de valorar los impactos de la variabilidad climática sobre la pesca y el turismo costero, para identificar si existe un marco metodológico integral que permita comparar estos procesos metodológicos y realizar un seguimiento para futuras valoraciones en estas áreas. En este caso, no se encontraron aproximaciones metodológicas que integren los diferentes pasos que se deben seguir para desarrollar la valoración económica de los diferentes impactos socioeconómicos y ambientales de este fenómeno tanto a nivel específico como a nivel global.
\end{abstract}

PALABRAS CLAVE: PESCA MARINA * TURISMO * VALORACIÓN ECONÓMICA * ZONAS COSTERAS * VARIABILIDAD CLIMÁTICA

\section{ABSTRACT}

The purpose of this article is to review the researches that have been developed to assess the impacts of climate variability on fisheries and coastal tourism, in order to identify whether there is an integral methodological framework to compare these methodological processes and to follow it for future ratings in these areas. Any methodological approaches that integrate the different steps to be followed for developing the economic assessment of different socio-economic and environmental impacts of climate change and climate variability both at specific and globally level, were not found.

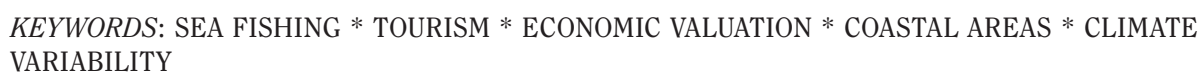

$1 \quad$ Este documento es parte del trabajo doctoral de la autora dentro del Doctorado en Ciencias Naturales para el Desarrollo (DocinadE), programa conjunto de la Universidad Nacional (unA-Costa Rica), Instituto Tecnológico de Costa Rica (TEC) y la Universidad Estatal a Distancia (UNED -Costa Rica).

Centro Internacional en Política Económica para el Desarrollo Sostenible (CINPE) de la Universidad Nacional (UNA), Costa Rica.

mmoreno@una.cr 


\section{INTRODUCCIÓN ${ }^{2}$}

El calentamiento del sistema climático es innegable, como lo evidencia el incremento de la temperatura media global del aire, la temperatura del océano, el derretimiento de nieve $y$ hielos, así como, el aumento global del nivel del mar (Intergovernmental Panel on Climate Change-IPcc, 2007). Las poblaciones y ecosistemas ubicados en las zonas costeras están experimentando las consecuencias adversas de los impactos relacionados con este calentamiento. Estas zonas albergan gran cantidad de riqueza biológica, económica y cultural, asimismo, son las áreas más productivas y biodiversas de los mares (el 90\% de los desembarques pesqueros proviene de allí) (Moreno, 2009). Además, se estima que $23 \%$ de la población del mundo vive dentro de los 100 kilómetros de la costa $y$ menos de 100 metros arriba del nivel del mar; las densidades de las poblaciones en las zonas costeras son cerca de tres veces mayores que el porcentaje global (Nicholls et ál., 2007).

Los efectos del cambio climático en las zonas costeras no fueron muy evidentes antes de 1750 (Nicholls et al., 2007); sin embargo, en los últimos años se ha evidenciado el aumento en la intensidad de los ciclones, aumento en el nivel del mar (Holgate y Woodworth, 2004) $y$ la temperatura superficial del mar ha aumentado en $0,6{ }^{\circ} \mathrm{C}$ desde 1950 (Bindoff et ál., 2007). Todo lo anterior tiene implicaciones sobre las comunidades, las instituciones y las organizaciones sociales costeras; por ejemplo, el hecho de que el nivel del mar de la costa está aumentando más rápidamente que el promedio, para la segunda mitad del siglo 20, tiene repercusiones significativas para los planificadores costeros (Holgate y Woodworth, 2004), en aspectos como la re-ubicación de comunidades que pudieran verse afectadas por este aumento.

$2 \quad$ La autora desea expresar su agradecimiento al Dr. Juan Camilo Cárdenas, profesor de la Escuela de Economía de la Universidad de los Andes (Colombia) y el Dr. Francisco Alpizar del Centro Agronómico Tropical de Investigación y Enseñanza-CATIE (Costa Rica) por su apoyo en el desarrollo de las pasantías que generaron parte de este documento.
Uno de los grandes retos de los especialistas en Valoración Económica es estructurar procesos metodológicos adecuados para analizar temas nuevos que son vitales para el desarrollo económico y social, como lo son el cambio y la variabilidad climática, así como, su impacto en los recursos naturales y sociales en las zonas costeras. Aunque no existe una metodología de valoración económica que se pueda aplicar a todo, ya que va a depender del problema a analizar, se debe seguir un proceso metodológico previo y durante la aplicación de la metodología de valoración, la cual permitirá recabar, ordenar y analizar la información necesaria para tal fin. Estos procesos metodológicos deben ser integrales (incorporar variables de ciencias naturales, sociales y económicas) $y$ ser replicables, tanto a nivel de actividades económicas específicas como a nivel global y así permitir futuras nuevas aplicaciones para un ámbito parecido; es decir, problemas relacionados con cambio o variabilidad climática, valoración socio-económica y zonas costeras.

El objetivo del presente artículo es analizar las investigaciones que se han desarrollado en los últimos años, tanto a nivel mundial como en los países de América Latina, para valorar los impactos del cambio y la variabilidad climática sobre la pesca y el turismo costero, con el fin de identificar si existe un marco metodológico integral que permita comparar estos procesos metodológicos, integrando variables de ciencias naturales, sociales y económicas.

\section{VALORACIÓN ECONÓMICA DEL CAMBIO CLIMÁTICO}

Una gran parte de las investigaciones relacionadas con el impacto económico de los efectos del cambio climático, han estado enfocadas en las metodologías que se basan en los costos. En este sentido:

La evidencia muestra gran diversidad de resultados, debido principalmente al uso de distintas metodologías y proyecciones climáticas, por adoptar distintos supuestos sobre la trayectoria de crecimiento económico y por enfocar distintos sectores, regiones o países. No obstante, 
hay consenso sobre la tendencia ascendente de los costos esperados, ya sea que se definan como pérdidas de bienestar o como proporción del pів (Comisión Económica para América Latina y el Caribe-CEPAL, 2011).

Los métodos para la estimación de costos directos de los efectos del cambio climático son relativamente simples de elaborar y fáciles de explicar. Básicamente, se estiman los costos incurridos en reparar y evitar las pérdidas ocasionadas por la intensificación de fenómenos climáticos (huracanes, tormentas, etc.) debido al cambio climático. Sin embargo, algunos de estos cálculos no tienen en cuenta modelos de emisiones ni climáticos, por lo tanto, no se puede asegurar que los impactos evaluados sean realmente producidos por el cambio climático y pueden deberse a variabilidades naturales del clima o a que las poblaciones se establecen en zonas altamente vulnerables (incremento de la vulnerabilidad por factores socioeconómicos) (Raschky, 2008).

Este tipo de estimaciones son cada vez más elaboradas, algunas relacionadas con el aumento en el nivel del mar que consideran pérdidas de tierra $y$ humedales, desplazamiento de poblaciones y protección costera con la construcción de diques (Tol, 2007). Algunos cálculos obtenidos de esta manera son: a) entre 1980 y 2005 , los Estados Unidos sufrieron 67 desastres relacionados con el clima, cada uno con un costo total de por lo menos US\$1 billón (National Climatic Data Center-scDc, 2007), b) las ciudades costeras del sur-este de los Estados Unidos tuvieron costos por Us $\$ 500$ billones en el período 1980-2002 (NcDc, 2007), c) en el 2001, los gastos anuales de protección de la línea costera en Europa se estimó en US\$4 billones (Nicholls et ál., 2007) y d) el costo promedio anual de restauración por ciclones tropicales en Japón entre 1950 y 2004, fue de us\$250 millones (Nicholls et ál., 2007).

Otro grupo de metodologías que se han empleado son aquellas que contienen variables macroeconómicas como las matrices insumo producto y los modelos de equilibrio general. Estas metodologías y herramientas se han desarrollado adecuándolas a la realidad de cada país en cuanto a pobreza, estado de los recursos naturales, situación económica, la disponibilidad de recursos financieros, humanos y técnicos (Nicholls et ál., 2007).

Los Modelos de Equilibrio General se han empleado para considerar mercados para todos los bienes y servicios simultáneamente, tomando en cuenta el comercio internacional y la inversión (Bosello, Lazzarin y Tol, 2007). Existen algunos modelos que incluyen también una representación de los procesos físicos y las restricciones (Nicholls et ál., 2007). Bosello et ál (2007) plantean un modelo en el que las pérdidas de tierra ocasionada por el aumento del nivel del mar $y$ los costos de protección costera, se calculan y luego se combinan con escenarios de aumentos en el nivel del mar, crecimiento de la población y crecimiento económico desarrollados por el Intergovernmental Panel on Climate Change (IPCC) en 1992, esto produce estimaciones de costo directo como un aproximado del cambio en el bienestar de las personas. La pérdida de tierras y el costo en protección son colocados en el Modelo de Equilibrio General (The Global Trade Analysis Project GTAP-EF) para simular efectos de equilibrio general sobre la economía mundial.

Hallegatte (2008) desarrolla un modelo insumo-producto regional con el cual simula la respuesta de la economía de Luisiana a la llegada del Huracán Katrina a tierra. Los procesos económicos exacerban las pérdidas directas y los costes totales se estiman en $\$ 149$ billones, en donde las pérdidas directas se calculan en $\$ 107$ billones.

También se han desarrollado a nivel mundial algunos estudios en los que se emplea el Producto Interno Bruto (PIB), como indicador para realizar una valoración global de los posibles efectos del cambio climático. Estos estudios han analizado diferentes sectores como agricultura, agua, bosques, biodiversidad, energía, salud, migración, elevación del nivel de mar $y$ eventos extremos como huracanes: "las metodologías también son diversas, desde modelos de valorización integrada, como el Page 2002, modelos de equilibrio general computable y los de enfoque sectorial. Cada modelo tiene diversas características y es utilizado para distintos propósitos y conduce a distintos resultados" (cEPAL, 2011: 257). Así por 
ejemplo, el informe Stern (2007) evidenció que sería más barato evitar la pérdida (cerca del $1 \%$ de PIB) producida por el cambio climático que no actuar (cerca del 20\% de PIB). En el informe de CEPAL (2011), se obtuvo que para implementar un escenario de estabilización de emisiones de $\mathrm{CO}_{2}$ a un nivel objetivo de 550 partes por millón (ppm), los costos acumulados al 2100 en términos de PiB, podrían variar en un rango de 4,7\%.

Para Centroamérica, en el último estudio publicado por CEPAL (2011) se realiza un análisis de los impactos económicos del cambio climático en los sectores y ámbitos prioritarios como el agrícola, recursos hídricos, biodiversidad y eventos extremos:

Partiendo de los escenarios tendenciales de crecimiento económico, demográfico $y$ cambio de uso de tierra sin cambio climático se generan líneas base del crecimiento de esos sectores y ámbitos. Una vez definida la trayectoria, se identifican los impactos probables de las variables temperatura y precipitación. En una segunda fase de la diferencia entre ambas trayectorias estiman los costos por sector... El valor total del impacto se traduce en unidades monetarias. La forma de expresarlo es asumir que la diferencia es un flujo en el tiempo que puede ser medido en unidades monetarias de hoy a Valor Presente Neto (vpN) con una tasa de descuento, $y$ que su proporción respecto al pIB es el costo del cambio climático (cEPAL, 2011:264).

Si bien, este estudio $y$ otros semejantes a este, tienen en cuenta modelos de emisiones y climáticos, el empleo de una variable macroeconómica como el PIB para medir los costos del cambio climático, tampoco asegura que los impactos evaluados sean realmente producidos por el cambio climático $y$ pueden deberse a variables como precios de insumos y precios de los productos, entre otros. Además, el hecho de que sean modelos macroeconómicos no permite analizar efectos a nivel de actividades económicas específicas.
VALORACIÓN ECONÓMICA DE BIENES Y SERVICIOS COSTEROS EN EL CASO DEL TURISMO Y LA PESCA

En general, el turismo es una industria dependiente del clima y muchos destinos deben su popularidad a su clima agradable y a su belleza escénica (Amelung, Nicholls y Vilmer, 2009). Los ambientes costeros y marinos se encuentran entre las áreas más populares para la recreación al aire libre $y$ el turismo. Las zonas costeras también han sido identificadas como las más vulnerables al cambio climático, por ejemplo, como resultado de eventos extremos y el aumento en el nivel del mar.

En términos de la valoración del turismo costero, Pendleton (2009) define la diferencia entre "valor económico" de los servicios brindados por los ecosistemas costeros y el "impacto económico". El valor económico representa el valor que los visitantes le dan al lugar ubicado en la zona costera, más allá de que ellos deban pagar por el acceso a los recurso. El impacto económico se define como los ingresos producidos por los visitantes a la economía local a través de los gastos en que incurren en actividades relacionadas, como el acceso, equipo y bienes y servicios (hielo, carnadas, etc).

Pendleton (2009) presenta un resumen de los estudios desarrollados sobre valores económicos recreativos de los ecosistemas costeros en los Estados Unidos entre 1975 y 2005. Según estas fuentes, la literatura económica brinda cerca de 58 estudios en los que se valoran los servicios recreativos de las zonas costeras de los Estados Unidos.

En el cuadro 1, se puede apreciar que los valores de cada una de las actividades expresada por los consumidores tienen rangos muy diferentes. Esto va a depender mucho del tipo de método que se emplee, del origen del visitante $y$ época del año, entre otros aspectos. Las metodologías más empleadas para valorar estas actividades son el método de valoración contingente y el método del costo de viaje. 
CUADRO 1

ESTUDIOS SOBRE VALORACIÓN DE LOS SERVICIOS AMBIENTALES RECREATIVOS DE LAS ZONAS MARINO COSTERAS EN LOS ESTADOS UNIDOS

1975-2005

\begin{tabular}{lcll}
\hline \multicolumn{1}{c}{ ACTIVIDAD } & $\begin{array}{c}\text { NRO. DE } \\
\text { ESTUDIOS }\end{array}$ & RANGOS DE VALORES & METODOLOGÍAS DE VALORACIÓN \\
\hline Visita a las playas & 29 & $\begin{array}{l}\text { Desde us } \$ 0,07 / \text { viaje hasta us } \$ 70 / \text { día } \\
\text { de actividad }\end{array}$ & $\begin{array}{l}\text { Costo de Viaje, Valoración Contingente, } \\
\text { Modelos Aleatorios de Utilidad, Análisis } \\
\text { Conjunto. }\end{array}$ \\
\hline Pesca deportiva & 13 & Entre us $\$ 20$ y us $\$ 100$ & $\begin{array}{l}\text { Costo de Viaje, Valoración Contingente } \\
\text { y Modelos Aleatorios de Utilidad. }\end{array}$ \\
\hline $\begin{array}{l}\text { Buceo } \\
\text { Observación de vida }\end{array}$ & 11 & $\begin{array}{l}\text { Entre us } \$ 15 / \text { actividad y us } \$ 50 / \\
\text { actividad }\end{array}$ & Valoración Contingente \\
\hline silvestre marino-costera & 5 & Entre us $\$ 133$ y us $\$ 228$ & Valoración Contingente, Costo de Viaje. \\
\hline
\end{tabular}

Fuente: Pendleton, 2009.

Otros estudios valoran los efectos económicos de un cambio en los recursos naturales sobre una actividad productiva, tal es el caso de algunos países caribeños. En esta región, se ha resaltado la importancia de los arrecifes de coral como una fuente importante de ingresos para el turismo, la pesca y las industrias farmacéuticas, así como, el papel fundamental que desempeñan en la protección de las costas, debido a los daños por erosión, inundaciones $y$ tormentas.

Para realizar una aproximación socioeconómica al efecto que tiene sobre las actividades productivas un deterioro del ecosistema coralino, por un lado, se estructuró un marco metodológico que valora los flujos de ingresos (brutos y netos, según la disponibilidad de información) de algunas actividades que obtienen beneficios de la existencia de estos ecosistemas y por el otro - cuando la información lo permite - calcula la disponibilidad de pago de los usuarios directos de los recursos (consumidores) por medio de una función de demanda.

Las actividades evaluadas con el modelo son: a) turismo: las actividades que reciben beneficios de la existencia de estos ecosistemas coralinos son hospedaje, transporte de turistas, empresas de buceo, snorkeling, tours, pesca deportiva y cruceros, cuando la información lo permite también se toma en cuenta el excedente de los turistas; b) pesca: la metodología calcula los ingresos brutos o netos de la porción del sector que depende del ecosistema coralino; se valoran las ventas formales e informales de la pesca, así como, el valor agregado del procesamiento; c) protección de la línea costera: una versión adaptada de la técnica de costos evitados fue usada para estimar el valor del servicio de protección de estos ecosistemas a lo largo de segmentos de la costa. Esto implica la estimación de las probables pérdidas económicas (en valor de la propiedad) de una zona costera por un evento de tormenta dada, con y sin los arrecifes $y$ los manglares presentes. Esta diferencia representa los "daños evitados" debido a la presencia de los arrecifes y los manglares (wri, 2009). Esta metodología se aplicó en República Dominicana, Jamaica y Belice. En el cuadro 2 se presentan las metodologías específicas aplicada para cada caso. 
CUADRO 2

METODOLOGÍAS EMPLEADAS PARA CALCULAR LA CONTRIBUCIÓN ECONÓMICA DE LOS ARRECIFES DE CORAL EN REPUBLICA DOMINICANA, JAMAICA Y BELICE

\begin{tabular}{|c|c|c|c|c|c|}
\hline \multirow{2}{*}{ PAÍS } & \multicolumn{2}{|c|}{ TURISMO } & \multicolumn{2}{|c|}{ PESCA } & \multirow{2}{*}{ FUENTE } \\
\hline & ACTIVIDAD & METODOLOGÍA & ACTIVIDAD & METODOLOGÍA & \\
\hline $\begin{array}{l}\text { República } \\
\text { Dominicana }\end{array}$ & $\begin{array}{l}\text { Hoteles. Se analizaron } \\
\text { los ingresos de los hote- } \\
\text { les teniendo en cuenta } \\
\text { las características de las } \\
\text { playas cercanas y la ubi- } \\
\text { cación del hotel. }\end{array}$ & Precios hedónicos & $\begin{array}{l}\text { Se analizaron los } \\
\text { ingresos por desem- } \\
\text { barques de la pesca } \\
\text { asociada a los arreci- } \\
\text { fes coralinos. }\end{array}$ & Ingreso bruto & $\begin{array}{l}\text { Wielgus et } \\
\text { ál., } 2010\end{array}$ \\
\hline Jamaica & $\begin{array}{l}\text { Turistas. Pérdida de exce- } \\
\text { dente por deterioro en las } \\
\text { playas. }\end{array}$ & $\begin{array}{l}\text { Disponibilidad de } \\
\text { pago }\end{array}$ & $\begin{array}{l}\text { Pesca. Se analiza- } \\
\text { ron los ingresos por } \\
\text { desembarques de la } \\
\text { pesca asociada a los } \\
\text { arrecifes coralinos. }\end{array}$ & Ingreso bruto & $\begin{array}{l}\text { Maxam et } \\
\text { ál., } 2011\end{array}$ \\
\hline Belice & $\begin{array}{l}\text { Hoteles. Se analizaron los } \\
\text { ingresos brutos e impues- } \\
\text { tos de recreación marina, } \\
\text { así como los ingresos por } \\
\text { alojamiento y otros gastos } \\
\text { de los turistas en los días } \\
\text { dedicado al uso de las pla- } \\
\text { yas coralinas y arrecifes. }\end{array}$ & Ingreso bruto & $\begin{array}{l}\text { Se analizaron los } \\
\text { ingresos brutos de } \\
\text { las actividades de } \\
\text { pesca y transforma- } \\
\text { ción comercial. }\end{array}$ & Ingreso bruto & $\begin{array}{l}\text { Cooper et } \\
\text { ál., } 2009\end{array}$ \\
\hline
\end{tabular}

Fuente: Elaboración propia con base en Wielgus, Cooper, Torres y Burke, 2010; Maxam, Lyew-Ayee y McIntyre, 2011 y Cooper, Burke y Bood, 2009.

\section{VALORACIÓN ECONÓMICA DE BIENES Y SERVICIOS COSTEROS EN EL CASO DEL TURISMO Y LA PESCA INCLUYENDO CAMBIO Y VARIABILIDAD CLIMÁTICA}

Se han desarrollado pocos estudios que involucran estas cuatro áreas a la vez. WeiChun y Chi-Chung (2007) elaboraron un estudio para estimar el valor del impacto económico potencial del cambio climático sobre la trucha de Taiwán, que es una especie en peligro de extinción. En primera instancia los autores establecieron que esta especie no sobrevive con temperaturas del agua mayores a 18 grados. Basados en los escenarios climáticos de Taiwán, en los cuales se proyectan aumentos de temperatura entre $0,9-2,7^{\circ} \mathrm{C}$ y aumento en la precipitación de 0,6 mm/día, los autores calculan las diminuciones en la producción de trucha cuando los valores en las temperaturas, debido al cambio climático aumentan en $0,9{ }^{\circ} \mathrm{C}, 1,8^{\circ} \mathrm{C}$ y $2,7^{\circ} \mathrm{C}$ y la precipitación también aumenta en $0,6 \mathrm{~mm} /$ día, encontrando valores de 974,560 y 146 truchas.

Luego de esto, los autores aplicaron un modelo de Valoración Contingente de Doble Límite para calcular la disponibilidad de pago en relación con cambios en la población de trucha de Taiwán. Para prevenir una reducción en el stock promedio de trucha de 1612 a 974 , la disponibilidad media de pago por persona por año fue de us $\$ 16,22$ con una desviación estándar de us\$1,3. Para prevenir una reducción en la población media de la trucha de 1612 a 560, la disponibilidad media de pago por persona 
por año fue de us $\$ 25,72$ con una desviación estándar de us $\$ 0,8$. Para prevenir una reducción en la población media de la trucha de partir de 1612 a 146, la disponibilidad media de pago por persona por año fue de us $\$ 33,6$ con una desviación estándar de us $\$ 1,7$.

Scott, Jones y Konopek (2007), desarrollaron un estudio en el que valoran el impacto en la disponibilidad de pago de los turistas ante un cambio en la precipitación y la temperatura en el Parque Nacional Rocky Mountain en Colorado. Emplearon la metodología de Valoración Contingente con encuestas a turistas para obtener respuestas de disponibilidad de pago y alguna información sobre actividades recreativas. Los autores encontraron que la temperatura y la precipitación fueron significativas estadísticamente en la determinación de la disponibilidad de pago de los turistas. Se estimaron incrementos en los beneficios recreativos de $4,9 \%$ y $6,7 \%$ para dos escenarios de cambio en el clima.

Shaw and Loomis (2008), realizaron un estudio en el que consideran un marco económico que permite el modelado de los efectos directos e indirectos del cambio climático en los usuarios de los recursos de recreación, a través de los impactos sobre los recursos naturales de los que depende la recreación al aire libre. Asimismo, presentan un breve resumen de algunos resultados empíricos que analizan la sensibilidad de las actividades recreativas a las variaciones en el clima. Algunos resultados de estos estudios fueron que, con el aumento relativamente pequeño en la temperatura que probablemente genera el cambio climático, el número de personas que participan en algunas actividades recreativas al aire libre, como paseos en barco, golf $y$ actividades recreativas de playa aumenten de $14 \%$ a $36 \%$. Por otro lado, el número de personas que participan en otras actividades, principalmente, los deportes de nieve como el esquí, probablemente disminuirá.

Se espera que el impacto del cambio climático en el sector pesquero, se vea reflejado tanto en aquellas especies que se desarrollan en las zonas costeras como aquellas que se encuentran en alta mar o pelágicas como en Nueva Zelanda y Australia (Hennessy et ál.
2007). Algunos de esos impactos serán: cambios en las temperaturas del mar, corrientes, vientos, oferta de nutrientes, acidificación y lluvias. Se prevé que dentro de las variables biológicas se presenten impactos en: a) distribución y abundancia de aquellas especies impactadas, b) composición de las comunidades, y c) estructura $y$ dinámica de las comunidades incluyendo productividad. Con el aumento del nivel del mar aumenta la intrusión marina afectando las pesquerías costeras y los sitios que son criaderos para especies comerciales (Schallenberg, Hall y Burns, 2003).

$\mathrm{Al}$ igual que en el caso del turismo, son pocos los estudios que relacionan valoración económica, pesca y cambio climático o variabilidad climática. Cardona (2009) desarrolla un modelo bioeconómico para pesca industrial de camarón blanco en el Pacifico Colombiano, con el fin de evaluar el efecto del Fenómeno del Niño sobre las capturas de esa especie.

Para el análisis se emplea las variables climáticas de temperatura superficial del mar $y$ duración promedio de cada evento asociado al Fenómeno del Niño, que son introducidas en el modelo de Schaefer y Gordon-Schaefer, con el fin de estimar el Rendimiento Máximo Sostenible (RMs), el Rendimiento Máximo Económico (RME) y el Rendimiento Bajo Libre Acceso (LA).

El modelo econométrico se presenta en la ecuación 1:

$$
\begin{gathered}
\text { Captura }=\alpha_{0}+\alpha_{1} \text { esfuerzo }+\alpha_{2} \text { esfuerzo }^{2}+ \\
\alpha_{3} \text { Temp }+\alpha_{4} \text { meses }+\alpha_{5} \text { Tend }+\alpha_{6} \text { período }+\varepsilon
\end{gathered}
$$

Ecuación 1

Donde:

$$
\begin{aligned}
\text { esfuerzo } & =\text { esfuerzo pesquero } \\
\text { Temp } & =\text { temperatura superficial en la región } \\
& \text { Niño } 3 \\
\text { meses } & =\text { duración del evento del niño en } \\
& \text { meses } \\
\text { Tend } & =\text { tendencia } \\
\text { período } & =\text { periodo de tiempo (1957-1984 y } \\
& 1985-2007)
\end{aligned}
$$

La variable período fue introducida debido a la presencia de cambio estructural. 
El estudio obtuvo como resultados que la intensidad del fenómeno del niño tiene efectos negativos sobre las capturas, ya que cada grado centígrado que aumente la temperatura superficial promedio del mar, representa una disminución en las capturas para el presente y el año siguiente. La duración también tiene efectos sobre las capturas pero estos son positivos, se obtuvo que por cada mes más que se produzca el aumento en las temperaturas, las capturas se van a ver incrementadas ese mismo año. La autora también determinó que a partir de 1984, se inició una disminución en las capturas probablemente debido al deterioro del recurso por sobreexplotación del mismo (Cardona, 2009), con los consiguientes efectos sobre el ingreso de las comunidades pesqueras.

Habib (2010), realizó un trabajo para Bangladesh en donde evalúa los impactos económicos sobre el stock de recursos pesqueros en presencia de cambio climático. Este autor emplea los modelos de Schaefer y GordonSchaefer para calcular el RMS, el RME $y$ el LA, en respuesta a cambios en los parámetros biológicos $\mathrm{K}$ (capacidad de carga del ambiente) y $\mathrm{r}$ (tasa intrínseca de crecimiento poblacional) con escenarios de cambio climático.

En este estudio, el autor no incluyó directamente las variables climáticas pero especificó nueve escenarios (incluyendo el actual) en los que $\mathrm{K}$ y $\mathrm{r}$ varían por influencia de esas variables. Se analizaron disminuciones de $25 \%$ y $10 \%$ en K y r, así como, sus posibles impactos en los rendimientos (RMS, RME y LA) y en los beneficios. Como resultado se obtuvo grandes diferencias en el caso de las capturas en (RMS, RME $y$ LA), el esfuerzo correspondiente $y$ el nivel de beneficios mostrados en cuatro escenarios en los que los niveles de beneficio variaron entre $25 \%$ a $64 \%$ de los obtenidos en el rue del escenario base. Lo anterior, permite analizar que los resultados son muy sensibles a la estimación de parámetros en los que hay un alto nivel de incertidumbre involucrado, lo cual hace el modelo menos confiable.

Según Hannesson (2007), los temas relacionados con pesca derivados del cambio global son: productividad sobre las poblaciones de peces (aumentos de unas poblaciones y dismi- nución de otras) y cambios en los hábitat (desplazamiento de concentraciones de peces).

Arnason (2007) proporciona estimaciones del impacto de las poblaciones alteradas de peces debido al calentamiento global en las economías de Islandia y Groenlandia. El enfoque se basa en simulaciones estocásticas. Esto implica básicamente tres pasos: 1) la obtención de predicciones sobre el impacto del calentamiento global en las poblaciones de peces y la distribución de la probabilidad asociada (empleando pronósticos de biología marina); 2) estimar el papel del sector pesquero en las dos economías, con la ayuda de las técnicas econométricas modernas basadas en la teoría del crecimiento económico y los datos históricos; y 3) llevar a cabo simulaciones de Monte Carlo sobre la base del modelo especificado anteriormente $y$ las incertidumbres asociadas. El resultado de la Simulaciones de Monte Carlo consiste en un conjunto de rutas dinámicas para el PIв con un valor esperado y una distribución probabilística para cada año futuro. Los resultados indican que el impacto en las pesquerías del cambio climático en el PIB de Islandia será más positivo que negativo, pero no parecen ser de magnitud considerable en comparación a las tasas históricas de crecimiento económico y las fluctuaciones.

\section{CONCLUSIONES}

El cambio y la variabilidad climática son fenómenos que han demandado gran cantidad de esfuerzos académicos y científicos para plantear formas de asimilarlo sin que las actividades económicas $y$ humanas se vean perjudicadas por sus efectos.

En el análisis realizado en el presente artículo, no se encontró un marco metodológico que permita identificar tanto la relación entre variables de las diferentes ciencias que intervienen, como los pasos que se deben seguir en las etapas previas a la aplicación de la metodología de valoración seleccionada para el problema identificado. Se concluye también que la incorporación de metodologías para valorar el efecto socio-económico de estos fenómenos en las economías locales, nacionales, 
regionales $y$ mundiales ha sido o muy local o demasiado global.

Asimismo, se concluye que hay grandes diferencias en las metodologías y que se requiere estructurar un proceso metodológico que permita incorporar los conceptos teóricos de ciencias naturales, sociales y económicas en un mismo esquema que permita "conectar" los efectos del sistema climático sobre los recursos naturales y sobre los ingresos de las actividades económicas que dependen de estos recursos naturales.

El cambio y la variabilidad climática son fenómenos que se irán intensificando en el futuro. El abordaje desde un marco metodológico adecuado permitirá contar con información valiosa para conocer a qué son vulnerables social y económicamente las comunidades costeras y cuáles serían las posibles acciones de mitigación que deberían tomarse en cuenta.

\section{BIBLIOGRAFÍA}

LIBROS

Habib, Ahasan. Economic impacts of fish stock resources in the case of climate change. Saarbrücken, Alemania: Lambert academic publishing, 2010.

Pendleton, Linwood. The economic and market value of america's coasts and esturies. Washington DC, EEUU: Costal ocean values press, 2009.

Stern, Nicholas. The economics of climate change: the stern review. London, Inglaterra: Cambridge press, 2007.

\section{PUBLICACIONES PERIÓDICAS}

Amelung, Bas; Nicholls, Sarah y Vinner, David. "Implications of global climate change for tourism flows and seasonality". Journal of travel research 45 (3). Florida, EEUU. Travel and Tourism Research Association, 2009: 285-296.

Arnason, Ragnar. "Climate change and fisheries: assesing the economic impact in Iceland and Greeland". Natural resource modeling 20 (2). New Jersey, EEUU. Wiley periodicals inc, 2007: 163-197.
Bosello, Franceso; Lazzarin, Roberto y Tol, Richard. 2007. "Economy-wide estimates of the implications of climate change: Sea Level Rise". Environmental and Resource Economics 37 ( 3). New York, EEUU. Springer, 2007: 549-571.

Hallegatte, Stephane. "An adaptive regional input-output model and its application to the assessment of the economic cost of Katrina”. Risk analysis 28 (3). Virginia, EEUU. Society of risk analysis, 2008: 779-799.

Hannesson, Rögnvaldur. "Introduction to special issue. Economic effects of climate change on fisheries". Natural resource modeling 20 (2). New Jersey, EEUU. Wiley periodicals inc, 2007: 157-162.

Holgate, Simon y Woodworth, Philip. "Evidence for enhanced coastal sea level rise during the 1990s". Geophysical research letters 31 (7). Washington DC, EEUU: American geophysical union, 2004.

Moreno, Mary. "Propuesta metodológica para valorar el impacto de las actividades económicas en áreas costeras". Rev. Iberoamericana de economía ecológica 11. México. Centro de Investigaciones en Ecosistemas (CIEco), Universidad Nacional Autónoma de México (UNAM), 2009: 29-38

Raschky, Paul A. "Institutions and the losses from natural disasters". Natural hazards and earth system sciences 8 (4). Munich, Alemania. European Geosciences Union, 2008: 627-634.

Scott, Daniel; Jones, Brenda y Konopek, Jasmina. "Implications of climate and environmental change for naturebased tourism in the Canadian Rocky mountains: a case study of Waterton Lakes National Park". Tourism management 28 (2). Hamilton, Nueva Zelandia. Centre for Management Studies, University of Waikato, 2007: 570-579.

Shaw, Douglas y Loomis, John. "Frameworks for analyzing the economic effects of climate change on outdoor recreation". Climate Research 36 (3). Alemania. 
Inter-Research Science Center, 2008: 259-269.

Schallenberg, Marc; Hall, Catherine y Burns, Carolyn. "Consequences of climate-induced salinity increases on zooplankton abundance and diversity in coastal lakes". Marine Ecology Progress Series. Alemania. Inter-Research Science Center, 2003: 181-189.

Tol, Richard. "The double trade-off between adaptation and mitigation for sea level rise: an application of FUND". Mitigation and Adaptation Strategies for Global Change 12. New York, EEUU. Springer, 2007: 741-753.

Wei-Chun, Tseng y Chen, Chi-Chung. "Valuing the potential economic impact of climate change on the Taiwan trout". Ecological Economics 65 (2). Ámsterdam, Países Bajos. Elsevier, 2007: 282-291.

TESIS

Cardona, Diana. "Análisis bioeconómico de la pesca de camarón blanco en el Pacífico colombiano en presencia del Fenómeno El Niño". [Tesis de Magister en Economía]. Colombia: Universidad de los Andes, 2009.

\section{TEXTOS ELECTRÓNICOS}

Bindoff, Nathaniel; Willebrand, Jurgen; Artale, Vincenzo; Cazenave, Anny; Gregory, Jonathan; Gulev, Sergey; Hanawa, Kimio; LeQuéré, Corrine; Levitus, Sydney; Nojiri, Yukihiro; Shum, C.K; Talley Lynne y Unnikrishnan, Alakkat. "Observations: oceanic climate change and sea level". Climate Change 2007: The Physical Science Basis. Susan Solomon, Dahe Qin, Martin Manning, Melinda Marquis, Kristen Averyt, Melinda Tignorand $y$ Henry Leroy (eds.). Inglaterra. Cambridge University Press, 2007: 385-432. En: $<$ http://www.ipcc.ch/pdf/assessmentreport/ar4/wg1/ar4-wg1-chapter5.pdf> [consultado el 13 de agosto de 2013].

Cooper, Emily; Burke, Lauretta y Bood, Nadia. Coastal Capital: Belize. The Economic
Contribution of Belize's Coral Reefs and Mongroves. Washington DC, EEUU: World Resources Institute, 2009. En: <http://pdf.wri.org/coral_reefs methodology_2009.pdf $>$ [consultado el 10 de julio de 2013].

Comisión Económica para América Latina y el Caribe-CEPAL. "Valorización económica del impacto del cambio climático. Comisión Económica para América Latina". Reporte Técnico. CEPAL, 2011: 256-281. En: <http://www.cepal.org/publicaciones/ xml/5/43925/2011-29-Cambio_climatico_ RT-L1016-Cap_10web.pdf> [consultado el 13 de noviembre de 2013].

Hennessy, Kevin; Fitzharris, Lair; Bates, Bryson; Harvey, Nick; Howden, Mark; Hughes, Lesley; Salinger, Jim y Warrick, Richard. "Australia and New Zealand. Climate change 2007: impacts, adaptation and vulnerability". Intergovernmental Panel on Climate Change 2. Geneva, Suiza, 2007: 507-540. En: <http://www.ipcc.ch/pdf/assessmentreport/ar4/wg2/ar4-wg2-chapter11.pdf> [consultado el 13 de agosto de 2013].

Nicholls, Robert; Wong, Poh; Burkett; Virginia; Codignotto, Jorge; Hay, John; McLean, Roger; Ragoonaden, Sachooda $y$ Woodroffe, Colin. "Coastal systems and low-lying areas". Climate change 2007: impacts, adaptation and vulnerability. Martin Parry, Osvaldo Canziani, Jean Palutikof, Paul van der Linden y Clair Hanso (eds.). Inglaterra. Cambridge University Press, 2007: 317-347. En: $<$ http://www.ipcc.ch/pdf/assessmentreport/ar4/wg2/ar4-wg2-chapter6.pdf> [consultado el 13 de agosto de 2013].

Maxam, Ava; Lyew-Ayee, Parrisand y McIntyre, Karen. A classification of the protection provided by coral reef systems in Jamaica-utilizing GIS and oceanographic methods of analysis. Washington DC, EEUU: World Resources Institute, 2011. En: <http://pdf.wri.org/working_papers/ coastal_capital_jamaica_shoreline_ protection_MGI.pdf $>$ [consultado el $20 \mathrm{de}$ julio de 2013]. 
Pachauri, Rajendra y Reisinger, Andy (dir.). "Intergovernmental Panel on Climate Change-IPCc". Cambio climático 2007. Informe de Síntesis. Cuarto informe de evaluación del grupo intergubernamental de expertos sobre el cambio climático. Geneva, Suiza, 2007. En: <http://www.ipcc.ch/pdf/assessmentreport/ar4/syr/ar4_syr_sp.pdf > [consultado el 13 de agosto de 2013].

Wielgus, Jeffey; Cooper, Emily; Torres, Ruben y Burke, Lauretta. Coastal capital: Dominican Republic. Case studies on the economic value of coastal ecosystems in the Dominican Republic. Washington DC, EEUU: World Resources Institute, 2010. En: <http://reefcheck.org/PDFs/ coastal_capital_dominican_republic. pdf > [consultado el 20 de julio de 2013].
World Resources Institute-WRI. Value of Coral reefs \& mangroves in the caribbeaneconomic valuation methodology V3.0. Washington DC, EEUU: World Resources Institute, 2009. En: <http://pdf.wri.org/ coral_reefs_methodology_2009.pdf> [consultado el 13 de noviembre de 2013].

OTROS

National Climatic Data Center-NCDC. Billion dollar us weather disasters, 1980-2006. 2007. En: <http://www.ncdc.noaa.gov/oa/ reports/billionz.html> [consultado el 25 de agosto de 2013].

Fecha de ingreso: 10/01/2014 Fecha de aprobación: 14/07/2014 
